\title{
Characterizing tumor invasiveness of glioblastoma using multiparametric magnetic resonance imaging
}

\author{
Chao Li, MD, PhD, ${ }^{1,2}$ Shuo Wang, PhD, ${ }^{3}$ Jiun-Lin Yan, MD, PhD, ,4,5 Turid Torheim, PhD, ,,7 \\ Natalie R. Boonzaier, PhD, ${ }^{1,8}$ Rohitashwa Sinha, MRCS, ${ }^{1}$ Tomasz Matys, PhD, FRCR, \\ Florian Markowetz, PhD, ${ }^{6,7}$ and Stephen J. Price, PhD, FRCS 1,10
}

\begin{abstract}
${ }^{1}$ Cambridge Brain Tumor Imaging Laboratory, Division of Neurosurgery, Department of Clinical Neurosciences, ${ }^{3}$ Department of Radiology, ${ }^{6}$ Cancer Research UK Cambridge Institute, and ${ }^{10}$ Wolfson Brain Imaging Centre, Department of Clinical Neurosciences, University of Cambridge, United Kingdom; '2Department of Neurosurgery, Shanghai General Hospital, Shanghai Jiao Tong University School of Medicine, Shanghai, China; ' $D$ Department of Neurosurgery, Chang Gung Memorial Hospital, Keelung, Taiwan; ${ }^{5}$ Chang Gung University College of Medicine, Taoyuan, Taiwan; ${ }^{7}$ CRUK \& EPSRC Cancer Imaging Centre in Cambridge and Manchester, Cambridge; ${ }^{8}$ Developmental Imaging and Biophysics Section, Great Ormond Street Institute of Child Health, University College London; and ${ }^{\circ}$ Cancer Trials Unit, Department of Oncology, Addenbrooke's Hospital, Cambridge, United Kingdom
\end{abstract}

OBJECTIVE The objective of this study was to characterize the abnormalities revealed by diffusion tensor imaging (DTI) using MR spectroscopy (MRS) and perfusion imaging, and to evaluate the prognostic value of a proposed quantitative measure of tumor invasiveness by combining contrast-enhancing (CE) and DTI abnormalities in patients with glioblastoma.

METHODS Eighty-four patients with glioblastoma were recruited preoperatively. DTI was decomposed into isotropic (p) and anisotropic (q) components. The relative cerebral blood volume (rCBV) was calculated from the dynamic susceptibility contrast imaging. Values of $\mathrm{N}$-acetylaspartate, myoinositol, choline (Cho), lactate (Lac), and glutamate + glutamine $(\mathrm{Glx})$ were measured from multivoxel MRS and normalized as ratios to creatine (Cr). Tumor regions of interest (ROIs) were manually segmented from the CE T1-weighted (CE-ROI) and DTI-q (q-ROI) maps. Perfusion and metabolic characteristics of these ROls were measured and compared. The relative invasiveness coefficient (RIC) was calculated as a ratio of the characteristic radii of CE-ROI and q-ROI. The prognostic significance of RIC was tested using Kaplan-Meier and multivariate Cox regression analyses.

RESULTS The Cho/Cr, Lac/Cr, and GIx/Cr in $q-R O I$ were significantly higher than CE-ROI ( $p=0.004, p=0.005$, and $p$ $=0.007$, respectively). CE-ROI had significantly higher rCBV values than $q-R O I(p<0.001)$. A higher RIC was associated with worse survival in a multivariate overall survival (OS) model (hazard ratio [HR] 1.40,95\% confidence interval [Cl] $1.06-1.85, p=0.016$ ) and progression-free survival (PFS) model (HR 1.55, 95\% Cl 1.16-2.07, $p=0.003$ ). An RIC cutoff value of 0.89 significantly predicted shorter OS (median 384 vs 605 days, $p=0.002$ ) and PFS (median 244 vs 406 days, $p=0.001$ ).

CONCLUSIONS DTI-q abnormalities displayed higher tumor load and hypoxic signatures compared with CE abnormalities, whereas $C E$ regions potentially represented the tumor proliferation edge. Integrating the extents of invasion visualized by DTI-q and CE images into clinical practice may lead to improved treatment efficacy.

https://thejns.org/doi/abs/10.3171/2018.12.JNS182926

KEYWORDS glioblastoma; MRI; diffusion tensor imaging; magnetic resonance spectroscopy; prognosis; oncology; perfusion imaging 
$\mathrm{G}$ LIOBLASTOMAS are the most common malignant brain tumors in adults, characterized by dismal patient survival. Although maximal safe resection followed by radiotherapy with concomitant and adjuvant temozolomide chemoradiotherapy has improved patient outcomes, the median overall survival (OS) remains 12-15 months. ${ }^{3}$ Accumulating evidence suggests that aggressive resection can prolong patient survival. ${ }^{8,24}$ However, extended resection or escalating adjuvant radiation volume may subject the patient to a higher risk of neurological deficits. A more accurate targeting of the tumor with less risk of functional damage to patients is therefore crucial for treatment planning.

MRI is widely used in monitoring the disease and defining the tumor region. In current clinical practice, contrast-enhancing (CE) T1-weighted imaging is considered the gold standard for planning surgery and radiotherapy. Glioblastoma is characterized by its infiltration into the surrounding brain tissue. Thus, infiltrative tumor cells are well known beyond the $\mathrm{CE}$ region. ${ }^{19}$ Other conventional sequences, including T2-weighted imaging and FLAIR, are nonspecific in differentiating tumor invasion from peritumoral edema and treatment effects that cause white matter change. ${ }^{28}$

Many MRI methods have been developed that provide signals relating to pathological changes in the tumor. ${ }^{16}$ Diffusion tensor imaging (DTI) measures the magnitude and direction of water mobility and is sensitive to detecting tumor invasion that leads to the disruption of brain microstructure. ${ }^{15,23}$ Previous work has shown that the diffusion tensor can be decomposed into isotropic (DTI-p) and anisotropic (DTI-q) components. ${ }^{14}$ The resulting DTI-p and $-\mathrm{q}$ maps have potential in delineating tumor invasion. ${ }^{6,19}$ Image-guided biopsy studies have shown that DTI-q abnormalities represent regions of higher tumor burden. ${ }^{19}$ A recent retrospective study revealed that more extensive resection of DTI-q and CE abnormalities was associated with improved patient outcome, suggesting both abnormality areas could be clinically relevant. ${ }^{29}$

The first purpose of this study was to compare the pretreatment DTI-q and CE abnormalities using magnetic resonance spectroscopy (MRS) and perfusion imaging in treatment-naive patients. As DTI-q may represent a tumor region with high tumor load, we hypothesized that the DTI-q abnormality would represent a more hypoxic region resulting from the higher cellularity in this region. On the other hand, because contrast enhancement is associated with contrast agent leakage, we hypothesized that the $\mathrm{CE}$ abnormality may represent a region with more evidence of neovascularization, indicated by the aberrant perfusion.

The second purpose of this study was to evaluate the prognostic value of a quantitative measure of tumor invasiveness, in which we integrated both volumes of DTI-q and $\mathrm{CE}$ abnormalities. Various studies have shown that hypoxia may be a factor driving tumor progression. ${ }^{2}$ Furthermore, angiogenesis can be activated by hypoxia through a series of proangiogenic factors, leading to neovascularization during tumor expansion. ${ }^{4}$ Therefore, we hypothesized that combining both DTI-q and CE abnormalities could be of prognostic value.

\section{Methods}

\section{Patient Population}

Patients with a suspected diagnosis of supratentorial newly diagnosed glioblastoma were prospectively recruited from July 2010 to August 2015. All patients were required to have a good performance status (WHO performance status 0-1) before surgery. Patients who had a history of a previous brain tumor, cranial surgery, radiotherapy/chemotherapy, or contraindication for MRI were excluded. This study was approved by the Cambridgeshire 2 LREC Ethical Committee. Signed informed consent was obtained from each patient.

\section{Treatment and Response Assessment}

Neuronavigation (StealthStation, Medtronic) and 5-aminolevulinic acid (5-ALA) fluorescence were used to guide surgery, with other adjuvants (e.g., cortical and subcortical mapping) to allow maximal safe resection when appropriate. Extent of resection (EOR) was assessed according to the postoperative MRI within 72 hours and classified as complete resection of enhancing tumor, partial resection of enhancing tumor, or biopsy. ${ }^{27}$ Patient treatment response was evaluated according to the Response Assessment in Neuro-Oncology criteria, ${ }^{28}$ which incorporate clinical and radiological measurements for assessment. All MRI and histological data were collected prospectively. In some cases, pseudoprogression was suspected when new enhancement was observed within 12 weeks after radiotherapy. In these cases, the patient's treatment was continued, and true progression or pseudoprogression was determined retrospectively.

\section{Preoperative MRI Acquisition}

All MRI sequences were performed on a 3-T MRI system (Magnetron Trio; Siemens Healthcare) with a standard 12-channel receive-head coil. ${ }^{7}$ MRI sequences were acquired as follows: postcontrast T1-weighted sequence (TR/TE/TI 2300/2.98/900 msec, flip angle $9^{\circ}$, FOV $256 \times$ $240 \mathrm{~mm}, 176-208$ slices, no slice gap, and voxel size $1.0 \times$ $1.0 \times 1.0 \mathrm{~mm}$ ) after intravenous injection of $9 \mathrm{ml}$ of gadobutrol (1.0 mmol/ml, Bayer); T2-weighted sequence (TR/ TE 4840-5470/114 msec, refocusing pulse flip angle $150^{\circ}$, FOV $220 \times 165 \mathrm{~mm}, 23-26$ slices, 0.5-mm slice gap, and voxel size $0.7 \times 0.7 \times 5.0 \mathrm{~mm}$ ); and T2-weighted FLAIR (TR/TE/TI 7840-8420/95/2500 msec, refocusing pulse flip angle $150^{\circ}$, FOV $250 \times 200 \mathrm{~mm}, 27$ slices, $1-\mathrm{mm}$ slice gap, and voxel size $0.78125 \times 0.78125 \times 4.0 \mathrm{~mm}$ ). Perfusion-weighted imaging was acquired with a dynamic susceptibility contrast-enhancement (DSC) sequence (TR/ TE 1500/30 msec, flip angle 90 ${ }^{\circ}$, FOV $192 \times 192 \mathrm{~mm}$, 19 slices, $1.5-\mathrm{mm}$ slice gap, and voxel size $2.0 \times 2.0 \times 5.0$ $\mathrm{mm})$ with $9 \mathrm{ml}$ of gadobutrol $(1.0 \mathrm{mmol} / \mathrm{ml})$ followed by a 20-ml saline flush administered via a power injector at $5 \mathrm{ml} / \mathrm{sec}$. DTI was acquired before contrast imaging, using a single-shot echo-planar sequence (TR/TE 8300/98 msec, flip angle $90^{\circ}$, FOV $192 \times 192 \mathrm{~mm}, 63$ slices, no slice gap, and voxel size $2.0 \times 2.0 \times 2.0 \mathrm{~mm}$ ); inline apparent diffusion coefficient calculation was performed during DTI acquisition using b values of $0-1000 \mathrm{sec} / \mathrm{mm}^{2}$. Multivoxel 2D ${ }^{1} \mathrm{H}-\mathrm{MRS}$ chemical shift imaging (CSI) utilized 
a semi-LASER sequence (TR/TE 2000/30-35 msec, flip angle $90^{\circ}$, FOV $160 \times 160 \mathrm{~mm}$, and voxel size $10 \times 10$ $\times 15-20 \mathrm{~mm}$ ). Point-resolved spectroscopy excitation was selected to encompass a grid of 8 rows $\times 8$ columns on T2-weighted images.

\section{Imaging Processing}

All other sequences were coregistered to the T2weighted images, which were used to plan the CSI in each subject. The coregistration was performed using the linear image registration tool functions in Oxford Centre for Functional MRI of the Brain Software Library (FSL; version 5.0.0). ${ }^{22} \mathrm{DSC}$ processing and leakage correction were performed with the NordicICE software (NordicNeuroLab), in which the arterial input function was automatically defined. The relative cerebral blood volume (rCBV) maps were calculated. DTI images were processed using the diffusion toolbox in FSL, ${ }^{1}$ during which normalization and eddy current correction were performed. The decomposition of processed DTI images into isotropic components (p) and anisotropic components (q) was performed using a previously described method..$^{14}$

\section{Regions of Interest}

Tumor regions of interest (ROIs) were manually segmented on the coregistered CE T1-weighted and DTI-q images. The delineation was independently performed blinded to the outcome by the authors with fair agreement (Fig. 1) ${ }^{6,29}$ To compare the imaging characteristics, a Boolean subtraction of $C E$ and $p$ abnormalities was performed in MATLAB (MathWorks) to obtain the CE-ROI (without DTI-q abnormality) and the q-ROI (anisotropic ROI).

For each individual patient, normal-appearing white matter (NAWM) regions were drawn manually in the contralateral white matter as normal controls. Each voxel value in the ROIs was normalized by dividing it by the mean voxel value of the contralateral NAWM.

\section{Tumor Characteristic Radius}

To quantify the extent of irregular tumors in 3D space, the characteristic radius of ROIs was calculated using a previously reported method. ${ }^{10}$ Briefly, the smallest bounding ellipsoid was fitted to the whole tumor volume revealed by ROIs. The length of this ellipsoid's semimajor axis was used to measure the characteristic radius of tumor ROI. We then calculated the ratio of $\mathrm{CE}$ characteristic radius to DTI-q characteristic radius, as a relative invasiveness coefficient (RIC). A demonstration of the calculation of characteristic radius and RIC is provided (Fig. 2).

\section{Multivoxel MRS Processing}

CSI data were processed using LCModel (Provencher). ${ }^{7}$ All the concentrations of metabolites were calculated as a ratio to creatine $(\mathrm{Cr}){ }^{5}$ All relevant spectra from CSI voxels of interest were assessed for artifacts using previously described criteria. ${ }^{20}$ The values of the Cramer-Rao lower bounds were used to evaluate the quality and reliability of CSI data and values with standard deviations > $20 \%$ were discarded. ${ }^{20}$

Because the CSI was planned on T2 space and CSI im-
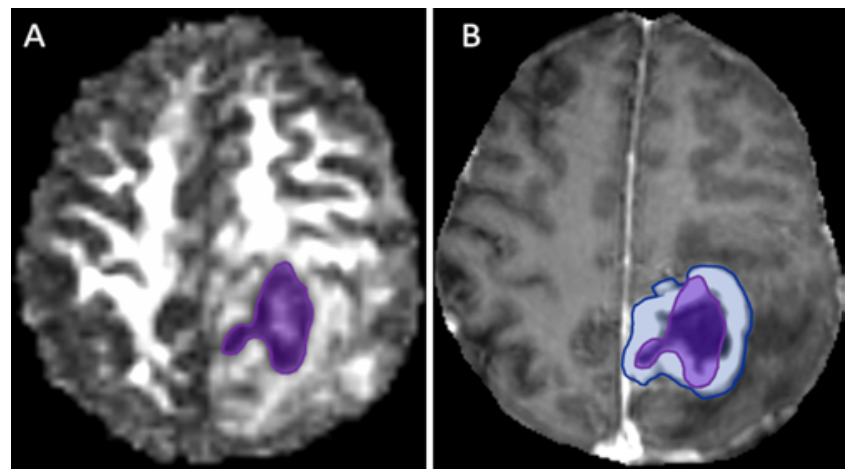

FIG. 1. A demonstration of ROls in a DTI-q image (A) and postcontrast T1-weighted image (B). The q-ROI (purple) shows the abnormality revealed on a DTI-q map. The CE-ROI (blue) is a CE tumor region excluding the DTI-q abnormality. Figure is available in color online only.

ages have lower spatial resolution than $\mathrm{T} 2$ images, to account for the difference in resolution, all tumor pixels in T2 space were projected to CSI space according to their coordinates using MATLAB. The proportion of T2-space tumor pixels occupying each CSI voxel was calculated. Only CSI voxels that were completely located within tumor ROIs were included for further analysis. The weight of each CSI voxel was taken as the proportion of the tumor pixels in that CSI voxel. The weighted sum value was used as the final metabolic value of the tumor ROIs.

\section{Statistical Analysis}

All statistical analyses were performed in RStudio (version 3.2.3). The CSI data and rCBV values were compared with pairwise Kruskal-Wallis rank-sum tests using the Benjamini-Hochberg procedure to control the false discovery rate in multiple comparisons. Categorical variables were tested using the chi-square test. The KaplanMeier and Cox proportional hazards regression analyses were performed to evaluate patient survival. In KaplanMeier analysis, RIC was dichotomized using the median value of all patients in the cohort. For Cox proportional hazards regression, all other relevant covariates, including isocitrate dehydrogenase 1 (IDH-1) mutation status, $O$ 6-methylguanine-DNA methyltransferase (MGMT) promoter methylation status, sex, age, EOR, and CE volume, were considered. Patients who were alive at the last known follow-up were censored. The hypothesis of no effect was rejected at a 2-sided level of 0.05 .

\section{Results \\ Patients}

A total of 136 patients were recruited. After surgery, 21 patients were excluded due to a nonglioblastoma pathology diagnosis. Due to their postoperative performance status, $84(73.0 \%)$ of 115 patients received concurrent temozolomide chemoradiotherapy followed by adjuvant temozolomide (Stupp protocol). Other patients received short-course radiotherapy $(17.4 \%, 20 / 115)$ or best supportive care $(9.6 \%, 11 / 115)$. We only included the 84 patients who received the Stupp protocol for final analysis. Survival data were available for $80(95.2 \%)$ of 84 patients and 

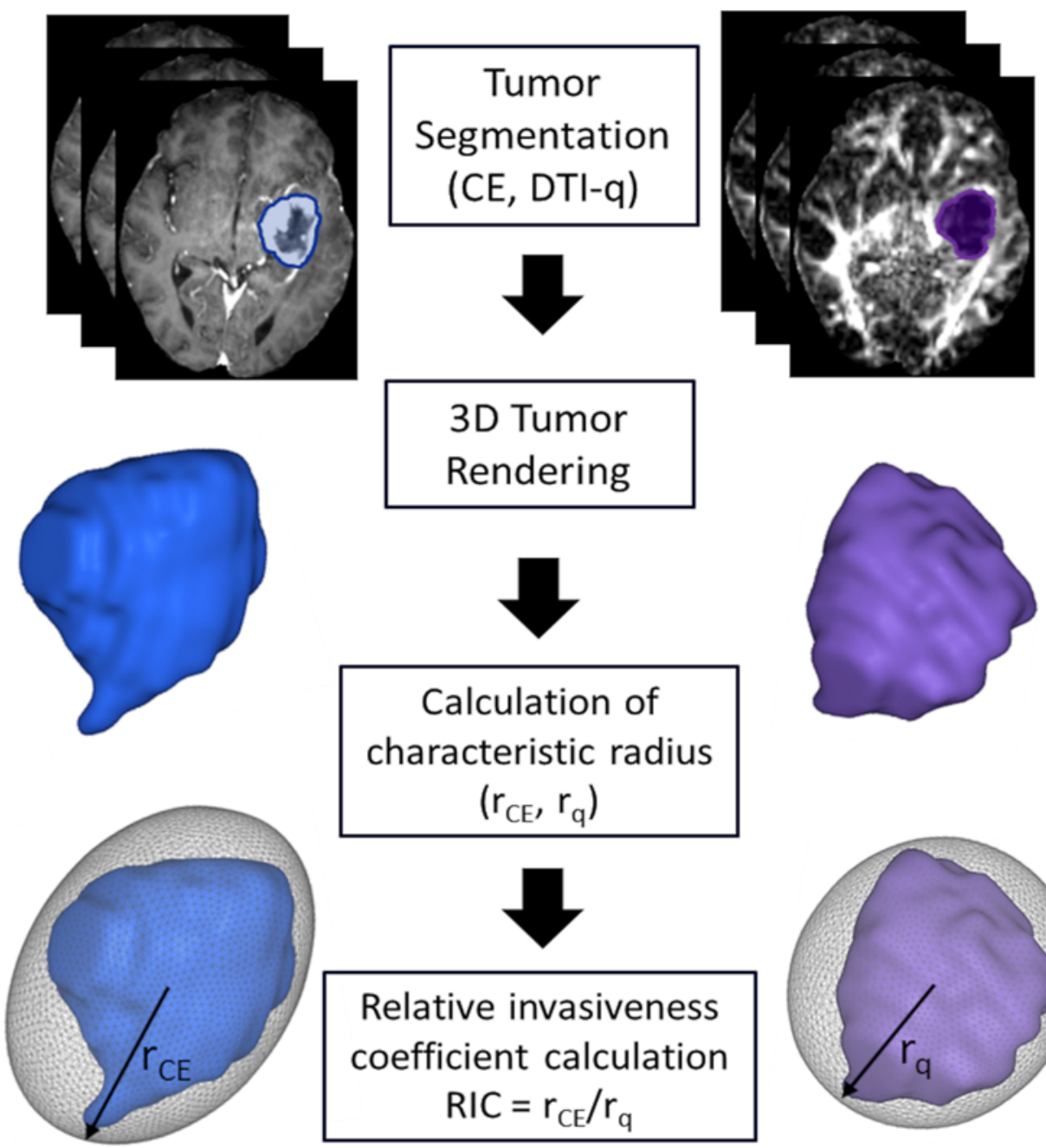

FIG. 2. A demonstration of calculation of the RIC. Tumor regions are segmented from postcontrast T1 (left) and DTI-q images (right), respectively. The smallest ellipsoids are fitted to the tumor volume. The characteristic radii of ROls $\left(r_{C E}, r_{q}\right)$ are calculated as the semimajor axis of ellipsoids. An RIC is calculated as the ratio of $\mathrm{r}_{\mathrm{CE}} / \mathrm{r}_{\mathrm{q}}$. Figure is available in color online only.

TABLE 1. Patient characteristics

\begin{tabular}{lc}
\hline \multicolumn{1}{c}{ Characteristic } & Value \\
\hline Total no. of patients & 84 \\
\hline Males, $\mathrm{n}$ & 62 \\
\hline Females, $\mathrm{n}$ & 22 \\
\hline Mean age \pm SD, yrs & $57.3 \pm 10.7$ \\
\hline Complete resection, $\mathrm{n}$ & 60 \\
\hline Partial resection, $\mathrm{n}$ & 22 \\
\hline Biopsy, $\mathrm{n}$ & 2 \\
\hline MGMT methylation positive, $\mathrm{n}(\%)^{*}$ & $37(45.1)$ \\
\hline IDH-1 mutation positive, $\mathrm{n}(\%)$ & $7(8.3)$ \\
\hline Mean CE vol \pm SD, $\mathrm{cm}^{3}$ & $44.2 \pm 27.2$ \\
\hline Mean DTI- $\mathrm{q}$ abnormality vol $\pm \mathrm{SD}, \mathrm{cm}^{3}$ & $43.8 \pm 27.1$ \\
\hline PFS (range), days & $264(25-1130)$ \\
\hline OS (range), days & $461(52-1376)$ \\
\hline
\end{tabular}

${ }^{*}$ MGMT methylation status unavailable for 2 patients.
4 patients $(4.8 \%)$ were lost to follow-up. Patient clinical characteristics are summarized in Table 1.

\section{Tumor Volume and Characteristic Radius}

The volumes of T1-weighted contrast-enhancement and DTI-q abnormalities are listed in Table 1. The mean $( \pm \mathrm{SD})$ characteristic radii of the $\mathrm{CE}$ tumor region and DTI-q abnormalities were $3.6 \pm 0.8 \mathrm{~cm}$ and $3.2 \pm 0.8 \mathrm{~cm}$, respectively.

\section{Imaging Characteristics of Tumor Regions}

In the comparison of rCBV, CE-ROI $(2.52 \pm 1.18,95 \%$ confidence interval [CI] 2.25-2.80) had significantly higher rCBV values than q-ROI $(2.08 \pm 1.23,95 \%$ CI $1.85-$ $2.30, \mathrm{p}<0.001)$. For MRS comparisons, both tumor ROIs displayed abnormal metabolic signatures compared to the NAWM (all $p<0.001$; Fig. 3). A full comparison is demonstrated in Supplementary Table 1.

Specifically, the choline/creatine $(\mathrm{Cho} / \mathrm{Cr})$ in $\mathrm{q}-\mathrm{ROI}$ $(0.66,95 \%$ CI $0.63-0.70)$ was significantly higher than in 

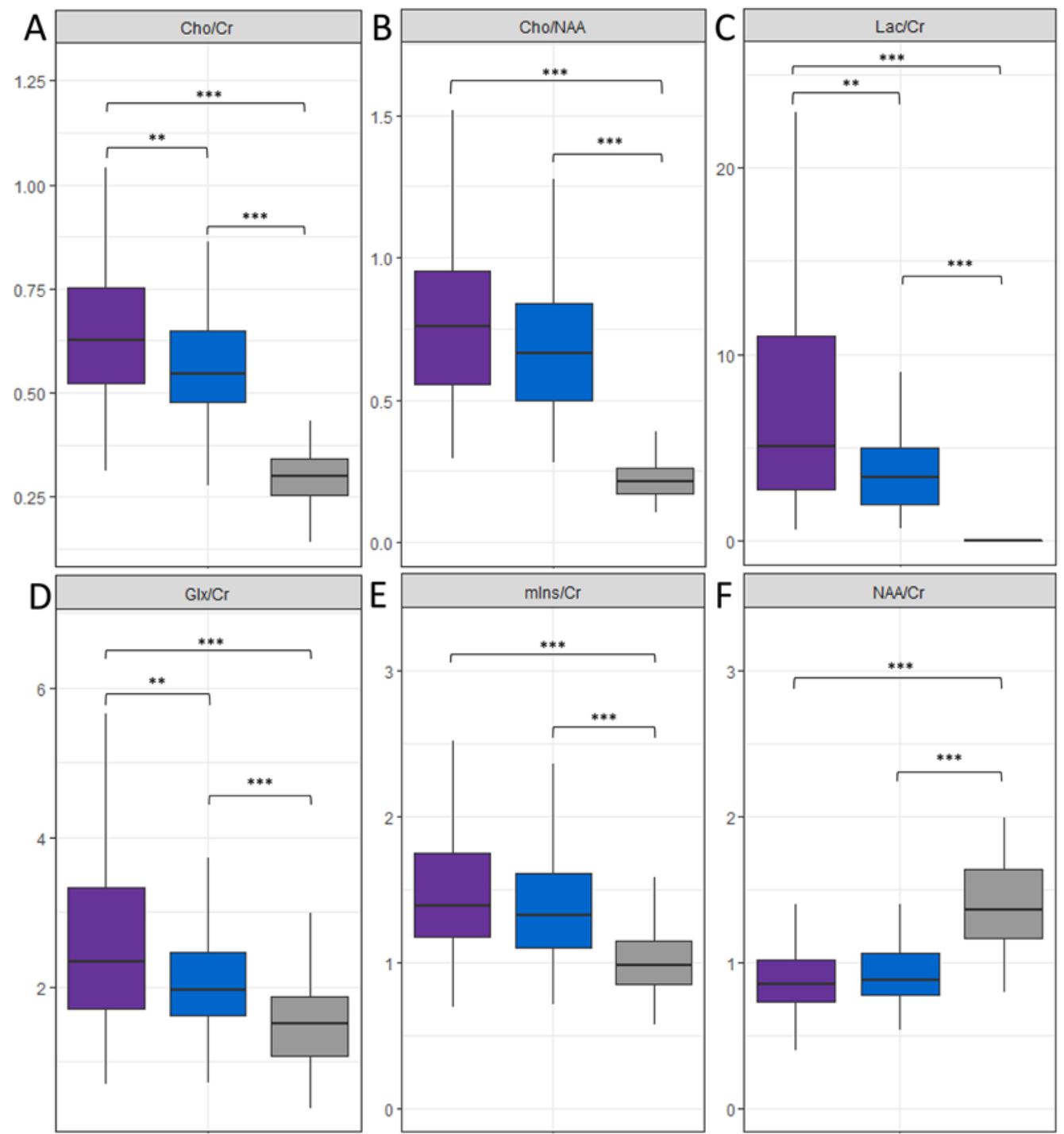

FIG. 3. Bar graphs of the metabolic signatures of ROls. Both tumor ROls display abnormal metabolic signatures. The q-ROI shows significantly higher levels of $\mathrm{Cho} / \mathrm{Cr}$, Lac/Cr, and $\mathrm{GIx} / \mathrm{Cr}$ than $\mathrm{CE}-\mathrm{ROI}$. ${ }^{* *} \mathrm{p}<0.01,{ }^{* *} \mathrm{p}<0.001$. Figure is available in color online only.

CE-ROI $(0.56,95 \%$ CI $0.54-0.59, \mathrm{p}=0.004)$. The lactate/ creatine $(\mathrm{Lac} / \mathrm{Cr})$ in q-ROI $(7.79,95 \%$ CI 6.34-9.24) was significantly higher than in CE-ROI $(4.17,95 \%$ CI $3.49-$ $4.85, \mathrm{p}=0.005)$. The glutamate + glutamine/creatine $(\mathrm{Glx} /$ $\mathrm{Cr})$ in q-ROI $(2.60,95 \%$ CI 2.373-2.83) was significantly higher than in CE-ROI $(2.08,95 \%$ CI $1.94-2.22, \mathrm{p}=$ $0.007)$.

Although not significant, the choline $/ N$-acetylaspartate (Cho/NAA) ratio of q-ROI $(0.84,95 \%$ CI $0.75-0.92)$ was higher than in CE-ROI $(0.75,95 \%$ CI $0.63-0.87, \mathrm{p}=$ $0.055)$. The myoinositol/creatine (mIns/Cr) ratio of q-ROI $(1.59,95 \%$ CI $1.44-1.73)$ was higher than in CE-ROI (1.45, $95 \%$ CI $1.33-1.56, p=0.085)$. There was no significant difference between the NAA/Cr ratios of q-ROI $(0.88$, 95\% CI 0.83-0.93) and CE-ROI (0.91, 95\% CI 0.86-0.95, $\mathrm{p}=0.171)$.

\section{Patient Outcomes}

The prognostic value of RIC was tested in multivariate survival models. The model of OS showed EOR (hazard ratio [HR] 2.32, 95\% CI 1.20-4.44, $\mathrm{p}=0.012$ ), the volume of CE (HR 1.59, 95\% CI 1.20-2.29, p = 0.007), and RIC (HR 1.40, 95\% CI 1.06-1.85, p = 0.016) significantly affected OS. In multivariate modeling of progressionfree survival (PFS), EOR (HR 3.27, 95\% CI 1.67-6.38, p $<0.001)$ and RIC (HR 1.55, 95\% CI 1.16-2.07, $\mathrm{p}=0.003$ ) significantly affected PFS.

We then dichotomized patients into 2 subgroups using the median value of RIC (0.89), with 42 patients in each group. The subgroup with an RIC $>0.89$ had a significantly shorter survival than those with an RIC $\leq$ 0.89 ( $p=0.001$ for PFS, $p=0.002$ for OS; Table 2, Fig. 4). No statistical difference was found between these 2 patient subgroups regarding age, sex, EOR, preoperative tumor volumes, or MGMT promoter methylation and IDH-1 mutation status (Table 2). Two case examples with an RIC $\leq 0.89$ and an RIC $>0.89$ are provided in Fig. 5. 
TABLE 2. Clinical characteristics of the patient subgroups

\begin{tabular}{|c|c|c|c|}
\hline Characteristic & $\mathrm{RIC}>0.89$ & $\mathrm{RIC} \leq 0.89$ & $p$ Value \\
\hline Mean age $\pm S D$, yrs & $58.1 \pm 10.3$ & $56.4 \pm 11.1$ & 0.661 \\
\hline Sex, $n$ & & & 0.457 \\
\hline Males & 33 & 29 & \\
\hline Females & 9 & 13 & \\
\hline EOR (of enhancing tumor) & & & 0.883 \\
\hline Complete & 29 & 30 & \\
\hline Partial & 12 & 10 & \\
\hline Biopsy & 1 & 1 & \\
\hline $\begin{array}{l}\text { MGMT promoter methyla- } \\
\text { tion status* }\end{array}$ & & & 0.183 \\
\hline Methylated & 15 & 22 & \\
\hline Unmethylated & 26 & 19 & \\
\hline IDH-1 mutation status & & & 0.430 \\
\hline Mutant & 2 & 5 & \\
\hline Wild-type & 40 & 37 & \\
\hline \multicolumn{4}{|l|}{$\begin{array}{l}\text { Mean preop tumor vol } \pm \\
\quad \mathrm{SD}, \mathrm{cm}^{3}\end{array}$} \\
\hline CE & $48.3 \pm 28.0$ & $40.0 \pm 14.7$ & 0.291 \\
\hline DTI-q & $41.5 \pm 27.4$ & $46.1 \pm 26.7$ & 0.263 \\
\hline \multicolumn{4}{|l|}{$\begin{array}{l}\text { Median survival (range), } \\
\text { days }\end{array}$} \\
\hline OS & $384(52-839)$ & $605(25-1130)$ & $0.002 \dagger$ \\
\hline PFS & $244(37-550)$ & $406(135-1259)$ & $0.001 \dagger$ \\
\hline
\end{tabular}

Boldface type indicates statistical significance.

${ }^{*}$ MGMT promoter methylation status unavailable for 2 patients.

† Log-rank test.

\section{Discussion}

In this study, we characterized CE and DTI-q abnormalities using multiparametric MRI and evaluated a proposed coefficient in assessing tumor invasiveness, which was calculated from the characteristic radii of $\mathrm{CE}$ and DTI-q abnormalities. The results showed that DTI-q abnormalities may represent a hypoxic tumor region. Integrating this coefficient into the survival model was useful in estimating tumor aggressiveness and predicting patient survival.

\section{DTI-q and Tumor Regions With Abnormal Metabolic Signatures}

Although providing useful structural information, conventional imaging is insufficient in reflecting tumor physiology ${ }^{19}$ The integration of advanced MRI techniques into clinical practice has been recommended for improving tumor volume targeting. Various findings support that DTI is useful in revealing tumor invasion and predicting patient survival..$^{9,18}$ In accordance with these studies, our current MRS results indicated that the q-ROI displayed abnormal metabolic signatures.

\section{DTI-q and CE Represent Different Tumor Properties}

In our results, the DTI-q abnormality showed a higher $\mathrm{Cho} / \mathrm{Cr}$ ratio than the $\mathrm{CE}$ region, which implies that the DTI-q abnormality regions may have higher cellularity than CE regions, as Cho is regarded as a marker of cell membrane turnover. ${ }^{12}$ The intensive tumor load in DTI-q abnormality may lead to hypoxia, which is supported by the significantly higher Lac/Cr level in DTI-q abnormality, compared to the CE region and normal control. The significantly elevated level of Glx, compared to the CE re-
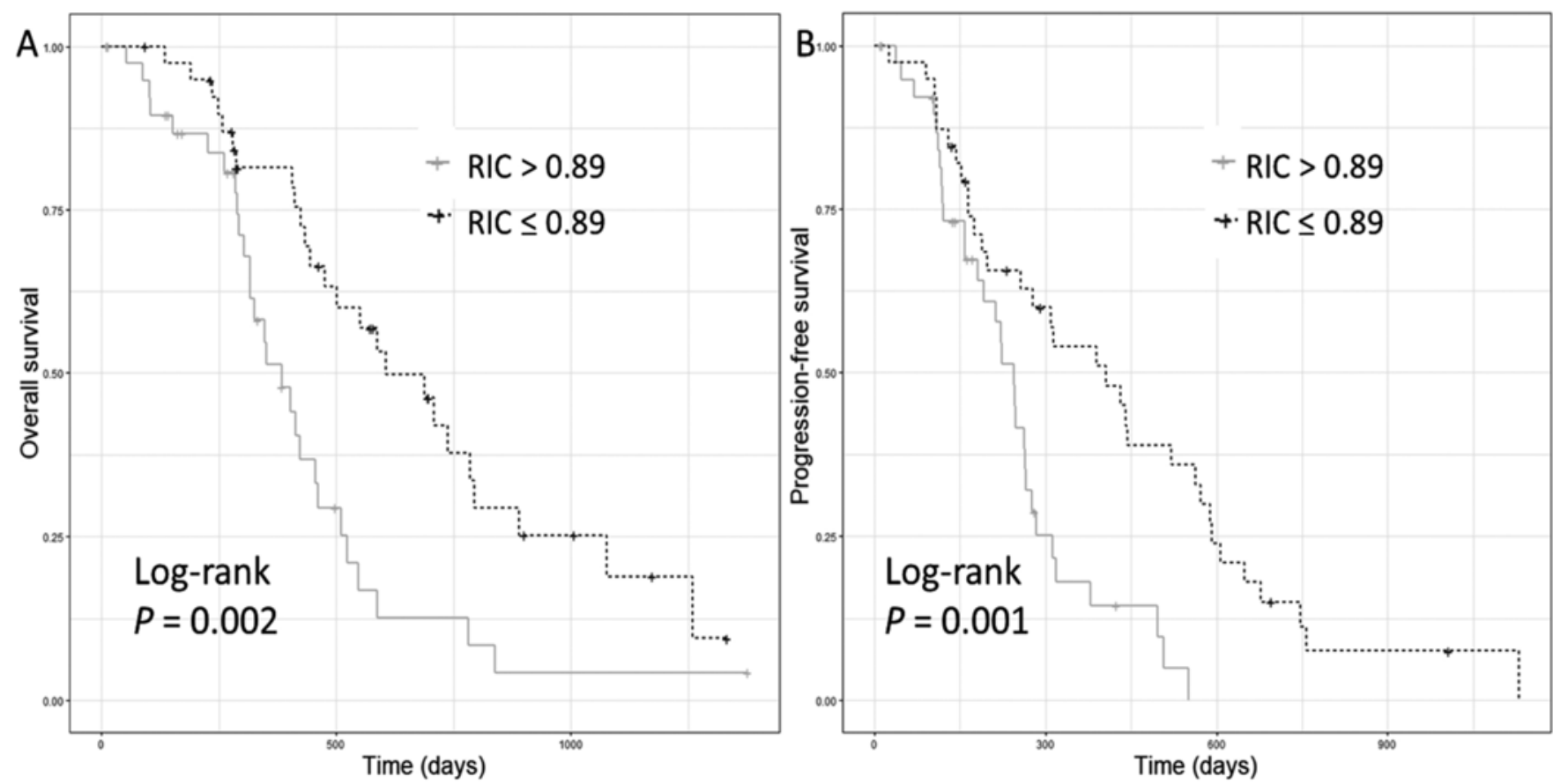

FIG. 4. Kaplan-Meier survival plots of patient clusters. The log-rank test showed patients with an RIC $>0.89$ displayed worse OS $(\mathbf{A}, p=0.002)$ and PFS $(B, p=0.001)$. 
gion, also suggested the neuronal destruction and hypoxic microenvironment in the DTI-q abnormality region. ${ }^{11,13}$ Compared to DTI-q abnormality, CE regions displayed a significantly elevated rCBV, which had a positive correlation with the MIB-1 index in a previous biopsy study. ${ }^{17}$ This suggests that the CE tumor may indicate a proliferative front of the tumor.

\section{RIC Combines DTI-q and CE Volumes}

It is well known that hypoxia may be a driving force of tumor invasion and proliferation. ${ }^{2}$ We hypothesized that the ratio of $\mathrm{CE}$ radius to DTI-q radius could indicate the tendency of tumor proliferation driven by hypoxia, which may potentially quantify tumor invasiveness. We tested this coefficient in the survival model and found clinical relevance with patient outcomes. Specifically, higher RIC may indicate a more aggressive tumor phenotype, in which the leading edge of proliferation runs further beyond the hypoxic core. It was previously reported that combining T1- and T2-weighted images is useful in quantifying tumor invasiveness. ${ }^{26}$ Here we proposed our approach by integrating physiological imaging with structural imaging, which might potentially provide a more physiologically relevant measure.

\section{Clinical Implications and Limitations}

Our study may have neurosurgical implications. An extended EOR can significantly improve OS of the newly diagnosed glioblastoma. ${ }^{21}$ The survival benefit from maximal safe resection is associated with not only extensive cytoreduction, ${ }^{25}$ but also improvement of the efficacy of a temozolomide regimen. ${ }^{24}$ Multiparametric MRI can facilitate the characterization of the multifaceted tumor properties. Our findings showed that DTI-q is an invasive region and that integrating CE and DTI-q volume may have prognostic value. Although the average volumes of CE and DTI-q abnormalities are similar, their locations and morphology can be different. In particular, for patients who have a greater extent of DTI-q abnormality than CE abnormality, a combination of both tumor areas into image-guided navigation may potentially bring benefits for maximal safe resection in surgical planning.

Our study had limitations. First, the resolution of CSI was lower than anatomical imaging and ${ }^{1} \mathrm{H}$ MRS voxels were larger than anatomical and physiological voxels. And second, our previous results have validated the imaging markers using histological assessment and longitudinal observations; further biological studies may be needed for future clinical application.

\section{Conclusions}

The imaging characteristics of DTI-q abnormality suggest that it is a hypoxic region with a high tumor burden. Integrating the extent of DTI-q and CE regions into clinical practice may bring benefits for patient care.

\section{Acknowledgments}

The research was supported by the National Institute for Health Research (NIHR) Brain Injury MedTech Co-operative based at Cambridge University Hospitals NHS Foundation Trust
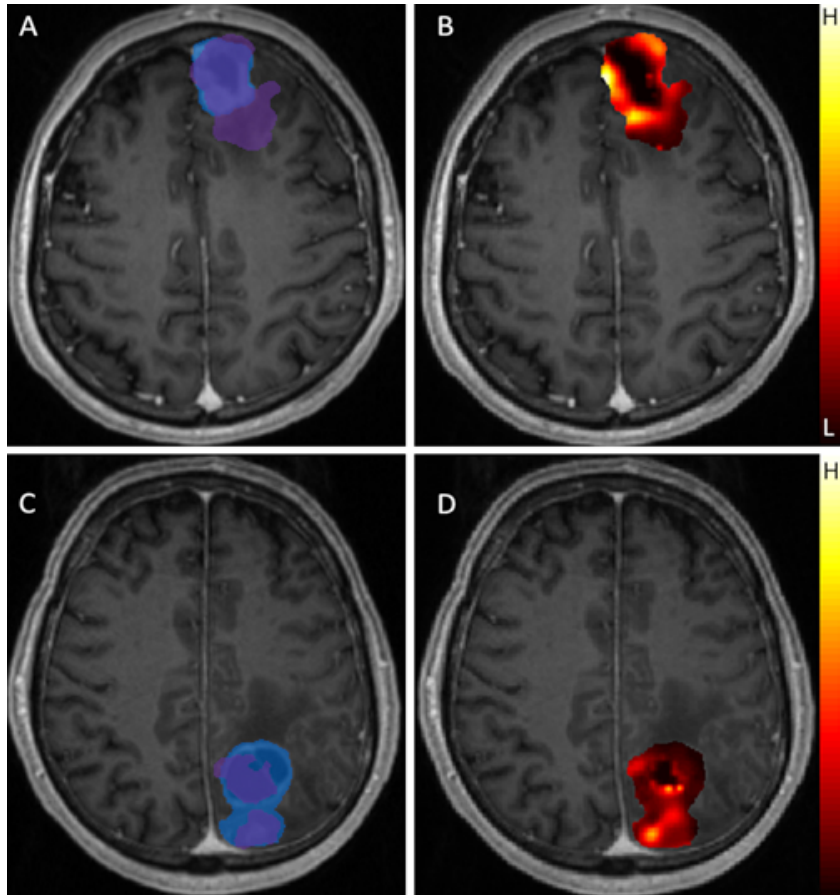

FIG. 5. Two case examples of tumor invasiveness revealed by CE tumor regions and DTI-q abnormalities. A and B: Case 1 is a 64-year-old man, with a CE tumor of $62.6 \mathrm{~cm}^{3}$ and RIC of 0.81 . C and D: Case 2 is a 69 -year-old man, with a CE tumor of $31.2 \mathrm{~cm}^{3}$ and RIC of 1.31. A and $\mathrm{C}$ indicate the CE-ROI (blue) and q-ROI (purple) in each case. B and D indicate the rCBV maps on a voxel-wise basis across the CE-ROI and $q-R O I$. Both patients received tumor resection with the guidance of neuronavigation and 5-ALA fluorescence for maximal resection. Complete resection was achieved in both patients according to the 72-hour postoperative MR images. Pathological assessment confirmed both were MGMT promoter unmethylated and IDH-1 wild-type glioblastomas. Both patients received concomitant and adjuvant temozolomide chemoradiotherapy following the Stupp protocol. The PFS and OS of case 1 were 563 and 697 days, respectively. The PFS and OS of case 2 were 159 and 317 days, respectively. Figure is available in color online only.

and University of Cambridge. The views expressed are those of the authors and not necessarily those of the NHS, the NIHR, or the Department of Health and Social Care (S.J.P., project reference NIHR/CS/009/011). In addition, this work was supported by a CRUK core grant (no. C14303/A17197 and A19274, F.M. laboratory); Cambridge Trust and China Scholarship Council (C.L. and S.W.); the Chang Gung Medical Foundation and Chang Gung Memorial Hospital, Keelung, Taiwan (J.L.Y.); CRUK \& EPSRC Cancer Imaging Centre in Cambridge and Manchester (F.M. and T.T., grant no. C197/A16465); the NIHR Cambridge Biomedical Research Centre (T.M. and S.J.P.); and the Commonwealth Scholarship Commission and Cambridge Commonwealth Trust (N.R.B.).

\section{References}

1. Behrens TE, Woolrich MW, Jenkinson M, Johansen-Berg H, Nunes RG, Clare S, et al: Characterization and propagation of uncertainty in diffusion-weighted MR imaging. Magn Reson Med 50:1077-1088, 2003

2. Colwell N, Larion M, Giles AJ, Seldomridge AN, Sizdahkhani S, Gilbert MR, et al: Hypoxia in the glioblastoma microenvironment: shaping the phenotype of cancer stem-like cells. Neuro Oncol 19:887-896, 2017

3. Hegi ME, Diserens AC, Gorlia T, Hamou MF, de Tribolet N, 
Weller M, et al: MGMT gene silencing and benefit from temozolomide in glioblastoma. N Engl J Med 352:997-1003, 2005

4. Kaur B, Khwaja FW, Severson EA, Matheny SL, Brat DJ, Van Meir EG: Hypoxia and the hypoxia-inducible-factor pathway in glioma growth and angiogenesis. Neuro Oncol 7:134-153, 2005

5. Kreis R: Issues of spectral quality in clinical ${ }^{1} \mathrm{H}$-magnetic resonance spectroscopy and a gallery of artifacts. NMR Biomed 17:361-381, 2004

6. Li C, Wang S, Yan JL, Piper RJ, Liu H, Torheim T, et al: Intratumoral heterogeneity of glioblastoma infiltration revealed by joint histogram analysis of diffusion tensor imaging. Neurosurgery [epub ahead of print], 2018

7. Li C, Yan JL, Torheim T, McLean MA, Boonzaier NR, Huang Y, et al: Low perfusion compartments in glioblastoma quantified by advanced magnetic resonance imaging: correlation with patient survival. bioRxiv [epub ahead of print], 2017

8. Li YM, Suki D, Hess K, Sawaya R: The influence of maximum safe resection of glioblastoma on survival in 1229 patients: can we do better than gross-total resection? J Neurosurg 124:977-988, 2016

9. Mohsen LA, Shi V, Jena R, Gillard JH, Price SJ: Diffusion tensor invasive phenotypes can predict progression-free survival in glioblastomas. Br J Neurosurg 27:436-441, 2013

10. Moshtagh N: Minimum Volume Enclosing Ellipsoid. (https://pdfs.semanticscholar.org/21c3/072e516c93b28ccd06f 5b994998abc517a7f.pdf) [Accessed February 25, 2019]

11. Noch E, Khalili K: Molecular mechanisms of necrosis in glioblastoma: the role of glutamate excitotoxicity. Cancer Biol Ther 8:1791-1797, 2009

12. Padhani AR, Miles KA: Multiparametric imaging of tumor response to therapy. Radiology 256:348-364, 2010

13. Pardon MC, Yanez Lopez M, Yuchun D, Marjańska M, Prior M, Brignell C, et al: Magnetic resonance spectroscopy discriminates the response to microglial stimulation of wild type and Alzheimer's disease models. Sci Rep 6:19880, 2016

14. Peña A, Green HAL, Carpenter TA, Price SJ, Pickard JD, Gillard JH: Enhanced visualization and quantification of magnetic resonance diffusion tensor imaging using the p:q tensor decomposition. Br J Radiol 79:101-109, 2006

15. Potgieser ARE, Wagemakers M, van Hulzen ALJ, de Jong BM, Hoving EW, Groen RJM: The role of diffusion tensor imaging in brain tumor surgery: a review of the literature. Clin Neurol Neurosurg 124:51-58, 2014

16. Price SJ, Gillard JH: Imaging biomarkers of brain tumour margin and tumour invasion. Br J Radiol 84:S159-S167, 2011

17. Price SJ, Green HAL, Dean AF, Joseph J, Hutchinson PJ, Gillard JH: Correlation of MR relative cerebral blood volume measurements with cellular density and proliferation in highgrade gliomas: an image-guided biopsy study. AJNR Am J Neuroradiol 32:501-506, 2011

18. Price SJ, Jena R, Burnet NG, Carpenter TA, Pickard JD, Gillard JH: Predicting patterns of glioma recurrence using diffusion tensor imaging. Eur Radiol 17:1675-1684, 2007

19. Price SJ, Jena R, Burnet NG, Hutchinson PJ, Dean AF, Peña A, et al: Improved delineation of glioma margins and regions of infiltration with the use of diffusion tensor imaging: an image-guided biopsy study. AJNR Am J Neuroradiol 27:1969-1974, 2006

20. Price SJ, Young AMH, Scotton WJ, Ching J, Mohsen LA, Boonzaier NR, et al: Multimodal MRI can identify perfusion and metabolic changes in the invasive margin of glioblastomas. J Magn Reson Imaging 43:487-494, 2016

21. Sanai N, Polley MY, McDermott MW, Parsa AT, Berger MS: An extent of resection threshold for newly diagnosed glioblastomas. J Neurosurg 115:3-8, 2011
22. Smith SM, Jenkinson M, Woolrich MW, Beckmann CF, Behrens TEJ, Johansen-Berg $\mathrm{H}$, et al: Advances in functional and structural MR image analysis and implementation as FSL. Neuroimage 23 (Suppl 1):S208-S219, 2004

23. Sternberg EJ, Lipton ML, Burns J: Utility of diffusion tensor imaging in evaluation of the peritumoral region in patients with primary and metastatic brain tumors. AJNR Am J Neuroradiol 35:439-444, 2014

24. Stummer W, Meinel T, Ewelt C, Martus P, Jakobs O, Felsberg J, et al: Prospective cohort study of radiotherapy with concomitant and adjuvant temozolomide chemotherapy for glioblastoma patients with no or minimal residual enhancing tumor load after surgery. J Neurooncol 108:89-97, 2012

25. Stummer W, Pichlmeier U, Meinel T, Wiestler OD, Zanella F, Reulen HJ: Fluorescence-guided surgery with 5-aminolevulinic acid for resection of malignant glioma: a randomised controlled multicentre phase III trial. Lancet Oncol 7:392401, 2006

26. Swanson KR, Rostomily RC, Alvord EC Jr: A mathematical modelling tool for predicting survival of individual patients following resection of glioblastoma: a proof of principle. $\mathbf{B r}$ J Cancer 98:113-119, 2008

27. Vogelbaum MA, Jost S, Aghi MK, Heimberger AB, Sampson JH, Wen PY, et al: Application of novel response/progression measures for surgically delivered therapies for gliomas: Response Assessment in Neuro-Oncology (RANO) Working Group. Neurosurgery 70:234-244, 2012

28. Wen PY, Macdonald DR, Reardon DA, Cloughesy TF, Sorensen AG, Galanis E, et al: Updated response assessment criteria for high-grade gliomas: response assessment in neuro-oncology working group. J Clin Oncol 28:1963-1972, 2010

29. Yan JL, van der Hoorn A, Larkin TJ, Boonzaier NR, Matys T, Price SJ: Extent of resection of peritumoral diffusion tensor imaging-detected abnormality as a predictor of survival in adult glioblastoma patients. J Neurosurg 126:234-241, 2017

\section{Disclosures}

The authors report no conflict of interest concerning the materials or methods used in this study or the findings specified in this paper.

\section{Author Contributions}

Conception and design: Li, Yan, Markowetz. Acquisition of data: Li, Yan, Boonzaier, Matys, Price. Analysis and interpretation of data: Li, Wang, Yan, Boonzaier. Drafting the article: Li, Yan, Sinha. Critically revising the article: Li, Wang, Boonzaier, Torheim, Sinha, Matys, Price. Reviewed submitted version of manuscript: Li, Wang, Boonzaier, Torheim, Sinha, Matys, Price. Approved the final version of the manuscript on behalf of all authors: Li. Statistical analysis: Li, Wang, Yan, Boonzaier, Torheim, Markowetz. Administrative/technical/material support: Li, Torheim, Markowetz. Study supervision: Markowetz, Price.

\section{Online-Only Content}

Supplemental material is available with the online version of the article.

Supplementary Table 1. https://thejns.org/doi/suppl/10.3171/ 2019.2.JNS182620.

\section{Correspondence}

Chao Li: University of Cambridge, United Kingdom.cl109@ outlook.com. 